\title{
Aller vers la dissolution : hybridité, ambivalence et monstruosité chez l'écrivaine mauricienne Ananda Devi
}

\author{
Markus Arnold \\ École supérieure d'Art de La Réunion
}

\begin{abstract}
Au matin, je tente de retenir la forme des monstres pour les restituer dans mes textes. Mais mes écrits contiennent des monstres à forme humaine. Ceux de mes rêves ont d'étranges excroissances ..., ce sont des abominations qui ... m'entraînent dans des tréfonds .... Que je sois proie ou prédateur, la sensation est la même. (Devi, Les hommes 168)
\end{abstract}

Depuis la fin des années 1990, la fiction romanesque mauricienne - du moins celle écrite en français et éditée en dehors de l'île — se distingue par des thématiques et poétiques violentes, par la déconstruction du mythe des beaux tropiques, et une tendance à l'intimité (narrateurs à la première personne, forte focalisation interne, flux de conscience...). Ces textes constituent une réelle rupture postcoloniale qui s'éloigne des perspectives représentationnelles et identitaires plus conventionnelles (à Maurice et des littératures émergentes plus généralement) et mettent en scène un univers insulaire qui se présente au lecteur tantôt comme opaque et bizarre, tantôt comme hideux, voire hostile. Cette anthropologie négative véhiculée par la fiction littéraire est souvent couplée d'un engagement contre des sectarismes et verrouillages identitaires d'une société traditionnelle et en faveur d'appartenances et de solidarités plurielles, hétérogènes et cosmopolites. En effet, malgré d'évidents phénomènes de créolisation et de multiplicité identitaire dans la jeune nation postcoloniale, le paradigme socio-ethnique officiel reste souvent limité à l'image d'un multiculturalisme rigide et schématique, ce à quoi de nombreux écrivains s'opposent.

Les propositions littéraires novatrices et progressives à Maurice témoignent donc souvent d'une certaine dés-exotisation ou «antitropicalisation» (Arnold «Les univers») qui se fait par l'usage d'un imaginaire étrange et difficilement compréhensible qui déréalise le lieu et ses habitants. Cet imaginaire rappelle en effet la notion de déréalisation qui, dans le domaine de la psychiatrie, se réfère à l'aliénation qui résulte d'une perception altérée du monde où personnes et objets perdent leur forme habituelle, paraissent fluides, démesurés, artificiels ou 'anormaux' (Piéron 116, Bloch et al. 331). Dans le texte littéraire, il s'agit d'une esthétique qui crée des effets de lecture inhabituels et qui tend à échapper aux logiques d'un réalisme mimétique. On retrouve donc dans plusieurs œuvres mauriciennes un indéniable sentiment de décalage et d'étrangeté qui ne s'explique pas par un

\footnotetext{
${ }^{1} \mathrm{Si}$ le français est la langue d'expression littéraire privilégiée à Maurice, il y a des auteurs qui écrivent en anglais, créole, voire en hindi, chinois et tamoul. De plus, l'on peut constater un positionnement artistique et identitaire, une esthétique, voire un style différents entre ceux édités localement et ceux édités à l'étranger.
} 
imaginaire culturel ou mythico-religieux quelconque. Des éléments insolites, oniriques et invraisemblables ainsi que des figures 'irréelles', 'anormales' et monstrueuses cohabitent avec un espace-temps insulaire bien reconnaissable. Métamorphoses, objets surnaturels et espaces liminaux font autant l'apparition que des personnages hybrides et des êtres interstitiels à la frontière entre l'humain et l'animal. Le monde parait à travers le prisme de la démesure, de l'aliénation, du fantasmagorique, de l'anthropomorphisme ; le réel se retrouve tantôt détourné, éclaté ou « dissolu» (Magdelaine-Andrianjafitrimo «J'ai préféré $»)$.

Cependant, une telle esthétique ne saura guère correspondre à des catégories d'analyses comme le fantastique ou des formes de merveilleux des littératures de l'océan Indien. En ancrant l'intrigue dans un espace-temps mauricien bien 'réel', mais avec des éléments oniriques, 'surnaturels' et 'irréels', ces textes surprenants résistent à une interprétation facile. Cette 'décolonisation' des imaginaires permet alors aux auteurs mauriciens de se positionner de manière tout à fait innovante dans l'espace de production littéraire francophone.

Ananda Devi, auteure à la trajectoire diasporique particulière entre Maurice, l'Afrique et l'Europe (Galibert), est une des figures-clés de l'ouverture et de l'éclatement des imaginaires dans le texte mauricien contemporain (Arnold, La littérature ch. V). Cette romancière prolixe - dont l'œuvre a déjà donné lieu à des études monographiques (p.ex. Tyagi, Kistnareddy) - crée en effet un univers fictionnel tout à fait original et singulier, complexe et lourd de sens. Elle y joue fréquemment avec l'étrange, l'hybride, le monstrueux, l'insolite, les zones d'indiscernabilités, les identités floues. L'objectif du présent article est de relever les modalités de cette esthétique particulière tissée autour de ces tropes de l' 'anormal', de l'hybride et du monstrueux pour en analyser la signification. Une analyse du roman $L a$ Vie de Joséphin le fou (2003), précédée de quelques éléments de conceptualisation, montrera que les représentations déréalisées chez Devi, loin d'être la mise en scène de simples fantasmes et délires oniriques et sans s'expliquer par un quelconque recours au spirituel ou au religieux, constituent des réflexions complexes sur les réalités et les enjeux identitaires de Maurice et du monde postcolonial en particulier, et sur l'humain en général.

\section{Éléments de contextualisation}

La déconstruction des imaginaires, l'usage de personnages troublés et monstrueux, la perturbation des logiques d'un réalisme mimétique invitent à une contextualisation des figures de l'insolite et de l'étrange dans (les littératures de) l'océan Indien. Évoquer des notions telles que l'étrangeté, la déréalisation ou la monstruosité rappelle bien sûr le champ de la littérature fantastique. Or, comme le dit Bernard Terramorsi («Le fantastique»), appliquer ce genre européen dit de l'impensable et de l'irreprésentable aux littératures non occidentales appelle à caution.

Depuis la perspective occidentale, on a tendance à associer ces phénomènes 'anormaux', présences fantastiques, monstrueuses, miraculeuses et surnaturelles au passé ou surtout aux espaces extra-occidentaux, en raison de l'importance que peuvent y avoir des traditions, mythes, inscriptions religieuses, croyances populaires et contes. L'on pense aux images rapportées 
par l'ethno-anthropologie - gri-gri africains, vaudous haïtiens, derviches persans, chamans amérindiens, charmeurs de serpents indiens, jeteurs de sorts aborigènes...- qui font souvent partie intégrante dans le vécu des nombreuses populations tandis que le logos occidental se réserve le monopole du réel 'désenchanté'. Que cette épistémologie soi-disant 'neutre', et du coup prétendument 'universelle', soit aussi tributaire d'idéologie a été relevé, et la question de savoir «Qu'est-ce le réel ? » a trouvé son entrée conflictuelle dans les études postcoloniales, entre autres à travers les travaux de Dipesh Chakrabarty. Cependant, d'éventuelles manifestations du baroque, du fantastique, du merveilleux, du surnaturel ou du mythique dans les textes euroaméricains, s'inscrivent dans les littératures de l'imagination bien circonscrites (science fiction, surréalisme, fantasy...). La présence de fantômes, loupsgarous, vampires et d'autres éléments irrationnels nous indique vite qu'on dépasse le cadre de la réalité prétendue objective et explicable.

Selon Roger Bozzetto (26-28), le discours universitaire distingue deux catégories du surnaturel. D'une part, on observe l'omniprésence des mythes dans toutes les cultures, qu'il s'agisse des mythes à la base des grands textes religieux et épiques (Gilgamesh, Odyssée, Bible, Mahabaratha...) ou des contes, avec une porosité des frontières entre vivants et morts, hommes, animaux et créatures surnaturelles. Il s'agit d'un merveilleux global et intemporel avec un fonds très vaste. D'autre part, on relève le fantastique,en tant que genre né dans le contexte idéologique et socioculturel bien déterminé de l'Europe à la fin du XVIII ${ }^{\mathrm{e}}$. Il exprime une mélancolie par rapport à une identité perdue, et marque le deuil de cadres symboliques dans un monde à prétention rationnelle déterminé par le progrès scientifique et le développement capitalo-industriel. L'atmosphère négative du fantastique, ses situations narratives inattendues, ses chutes catastrophiques, son interruption brutale du chronotope réaliste laissent le héros et le lecteur devant une aporie intellectuelle et une incohérence irréductible qui sapent le rapport au monde et créent une angoisse ontologique. Le fantastique devenant ainsi un concept particulièrement pertinent pour la littérature occidentale, le fait de retrouver dans les cultures non européennes une prégnance de la tradition orale avec ses thématiques surnaturelles et sa pensée magique empêcherait donc l'essor du genre fantastique (Terramorsi, « Le fantastique » 171-172).

Si l'on peut alors difficilement parler de littérature fantastique dans l'océan Indien, que faire à partir du moment où le fantastique est bien présent dans ses cultures en tant que manière de penser et rapport au monde ? Car diables, fantômes, revenants, spectres, morts-vivants, âmes errantes, monstres, métamorphoses, enchantements, sorcelleries, superstitions sont en effet autant de catégories anthropologiques facilement repérables dans ces lieux insulaires. Le sacré n'y est aucunement mort et continue à donner un sens au monde. Les temporalités résistent à une fixation rigide et la thèse de Chakrabarty sur la nécessité de prendre en compte dans la production de savoir la pluralité temporelle et des éléments métaphysiques ${ }^{2}$, et d'interroger la logique du réalisme mimétique et sa pseudo-rationalité, trouve dans cette zone géographique et culturelle toute son application. En d'autres termes, une

\footnotetext{
2 « Historical time is not integral, ... it is out of joint with itself. ... I take gods and spirits to be existentially coeval with the human, and think from the assumption that the question of human beings involves the question of being with gods and spirits » (Chakrabarty 16).
} 
dissociation arbitraire entre nature et culture et entre matériel et immatériel y est impossible (Marimoutou 134), et l'ouvrage collectif Démons et merveilles. Le surnaturel dans l'océan Indien (2005) montre les multiples manifestations de l'élément fantastique et ses déclinaisons dans les littératures indianocéaniques.

L'enchevêtrement de différents niveaux de réalité d'une manière harmonieuse et sur le plan de l'évidence, qui reste incompatible avec le logos européen rappelle bien sûr le "real maravilloso", établi en 1949 par le Cubain Alejo Carpentier. Repris ultérieurement par le Haïtien JacquesStephen Alexis pour sa notion du "réalisme merveilleux », il exprime l'ancrage de la spécificité de l'imaginaire caribéen et latino-américain dans une tradition vivante du mythique, du légendaire, du magique. Le terme a une longévité certaine : les propositions de la Créolité antillaise y souscrivent ${ }^{3}$ et il peut aussi apparaître dans la littérature réunionnaise. On a même pu l'utiliser (abusivement) pour référer à tout auteur incluant dans ses textes narratifs du matériel mythique ou légendaire issu des traditions culturelles, comme c'est le cas chez des figures aussi diverses que Salman Rushdie, Ben Okri ou Keri Hulme (Ashcroft et al. 133). Cet embarras terminologique concerne aussi les littératures de l'océan Indien et Terramorsi parle alors d'un « réalisme indianocéanique », ou, en raison de son hétérogénéité, d'un « improbable fantastique indianocéanique » («Préface» 6, 8) pour des textes où l'on déterminera des éléments surnaturels, étranges, merveilleux ... sans pour autant pouvoir parler de genre ou de courant esthétique.

Il est évident que plusieurs romans de l'île Maurice souscrivent également à une telle esthétique ${ }^{4}$. Ils s'opposent souvent à la conception de phénomènes objectifs et de causalités rigoureuses, violent les lois naturelles, opèrent un mélange de réalisme mimétique et d'étrangeté irréaliste, déploient une rhétorique de l'indicible. Autrement dit, ils empruntent à l'esthétique du fantastique, sans entièrement y correspondre. Quant à la présence d'éléments mythologiques et d'autres références à un univers symbolique et traditionnel mauricien - cette « surnature codée et collectivement admise » (Terramorsi «Préface » 6) que sont les croyances ancestrales, les religions traditionnelles et le fonds légendaire-, de tels éléments peuvent certes apparaître. Or des phénomènes comme un certain "surnaturel indien " ou d'autres marqueurs culturels, mythiques et religieux ne sauraient expliquer la signification des processus de déréalisation mis en scène, chez certains auteurs, et notamment Devi. Car l'étrangeté, la déréalisation ou la monstruosité dans le cas de certains textes mauriciens opèrent une forme de déconstruction des représentations et de l'imaginaire littéraire très particulière qui a du mal à correspondre aux définitions convenues.

Devi fournit la seule définition émanant de l'océan Indien et de à ce qu'elle nomme le «para-naturel» dans une interview ainsi : «je décris une

\footnotetext{
3 « Notre écriture doit accepter sans partage nos croyances populaires, nos pratiques magicoreligieuses, notre réalisme merveilleux » (Bernabé et al. 40).

${ }^{4}$ A côté de textes de Devi - notamment Moi l'interdite (2000) et Pagli (2001) -, l'on pense par exemple aux Jours Kaya (2000) de Carl de Souza, Histoire d'Ashok et d'autres personnages de moindre importance (2001) et Les voyages et aventures de Sanjay, explorateur mauricien des Anciens Mondes (2009) d'Amal Sewtohul, de même qu'à des œuvres anglophones, telles que The Book of Colour (1995) de Julia Blackburn ou The Snake Spirit (2002) de Chaya Parmessur.
} 
situation et des personnages "réels", mais la ligne de démarcation avec une autre dimension de pensée et de conscience est très mince, de sorte que s'imbriquent souvent des situations réelles et "sur-réelles" »(Sultan). Qu'il s'agisse des métamorphoses brouillant les frontières entre l'humain et l'animal dans Moi l'interdite (2000), des femmes malveillantes et fantomatiques dites mofines dans Pagli (2001) ou bien des esprits entourant les personnages traqués dans Soupir (2002): les exemples de ce «para-naturel », toujours associé à un sentiment d'angoisse, ne manquent pas dans l'œuvre de l'écrivaine.

$\mathrm{Si}$ cette imbrication et cette fusion entre différents mondes et dimensions semblent venir «tout naturellement» (Sultan), —Devi étant de surcroît très influencée par son ascendance culturelle indienne- une telle écriture ne peut être rattachée à une vision du monde indien. Comme le dit Magdelaine-Andrianjafitrimo («Les égarements» 435), la création de la notion de «para-naturel»-qu'elle identifie chez Devi par des éléments particuliers tels que des métamorphoses, situations étranges, créatures animales et humaines hybrides éloignées du religieux, du spirituel, des mythes indianocéaniques - traduirait plutôt un ancrage de l'auteure dans une conception du réel 'à l'occidentale' duquel son écriture transgressive se dissocie. Conceptualisation floue et sans réel enracinement du texte littéraire dans les cultures traditionnelles, le «para-naturel» semble témoigner avant tout d'une «mythologie personnelle» de l'auteure, qui, comme elle le souligne dans son texte autobiographique Les hommes qui me parlent, " n'est pas gaie » (Devi 36). Ce positionnement traduit un rapport spécifique au monde qui reste plus d'une fois très hermétique dans ses productions littéraires.

\section{L'étrange univers littéraire d'Ananda Devi}

Depuis plusieurs années, Ananda Devi s'affirme comme l'une des figures majeures de la littérature de l'océan Indien. Auteure prolixe depuis son adolescence, son œuvre romanesque, poétique et intime a été remarquée par la critique $^{5}$ et a même donné lieu à des adaptations cinématographiques dans lesquelles 1'auteure était en partie impliquée ${ }^{6}$. Vivant depuis longtemps à l'étranger et revisitant son île natale en permanence à travers la distance, Devi est une des cosignataires du manifeste de la Littérature-monde en français ${ }^{7}$ et elle est depuis 2006 publiée dans la prestigieuse collection dite «Blanche » de Gallimard. Elle a été l'objet de divers travaux et manifestations de recherche thèses, monographies, colloques - et des numéros spéciaux de revues lui sont

\footnotetext{
${ }^{5}$ Entre autres, le Prix Radio France du Livre de l'Océan Indien pour Moi, l'interdite (2000), le Prix des Cinq Continents de la Francophonie et le Prix RFO du livre pour Ève de ses décombres (2006), le Prix Louis Guilloux pour Le sari vert (2009).

${ }^{6}$ En effet, les deux longs-métrages La Cathédrale (2006) et Les enfants de Troumaron (2012) -basés respectivement sur la nouvelle La Cathédrale (1977) et le roman Ève de ses décombres (2006) de Devi- ont été réalisés par son époux, le cinéaste mauricien Harrikrisna Anenden, et produits dans leur maison de production Cine Qua Non Ltd.

${ }^{7}$ Pour une littérature-monde. Éds. Michel Le Bris et Jean Rouaud. Paris: Gallimard, 2007.
} 
consacrés $^{8}$. En 2010, l'auteure est nommée au grade de chevalier dans l'ordre des Arts et des Lettres.

Son œuvre riche touche à la question de l'histoire et de la mémoire, de l'altérité, à la condition des subalternes et marginaux, aux mythes ancestraux - surtout le sacré indien-, à la souillure et la transgression, à la norme, la violence et la malédiction. Opposée violemment aux politiques multiculturalistes et aux dérives identitaires, Devi se prononce souvent en faveur de la multi-appartenance et des identités métisses. Dans ses ouvrages, une focalisation particulière revient sur l'identité féminine, la sororité, l'écriture du corps et ce que l'auteure elle-même appelle une "carnalité ", c'est-à-dire le réveil chez ses personnages d'une " animalité qui va jusqu'à la métamorphose physique » (Marson 64). Mais elle traite aussi de l'exploitation néocoloniale de la mondialisation contemporaine et propose, dans ses derniers textes, une ouverture extrainsulaire, s'inscrivant ici dans une tendance littéraire à Maurice où la nécessité de dire l'île pour se légitimer semble être dépassée. Enfin, le trait scripturaire le plus saillant chez l'auteure s'avère le caractère très poétique et viscéral de ses textes en prose ainsi qu'une indéniable amertume existentielle et l'anthropologie négative que cette esthétique véhicule. L'ambiguïté, l'étrangeté et le fantastique bien particulier qui habitent les textes de Devi ont pu être analysés par le prisme du « réalisme magique », d'une « forme de surnaturel », d'une « surnaturalité » (Ramharai), sans oublier au-delà de ces complications terminologiques, nous l'avons dit, le terme de « para-naturel », créé par l'auteure elle-même.

Chez Devi, le trope de la monstruosité prend une place aussi importante qu'elle l'évoque à plusieurs moments dans son récit autobiographique : qu'il s'agisse du fantasme de se débarrasser des marqueurs physiques du genre ${ }^{9}$, des objets cauchemardesques au symbolisme mortifère ${ }^{10}$, et, de manière plus générale, de la face cachée de son île qu'elle décrit ainsi : « un univers peuplé de monstres et de prisonniers » (Devi, Les hommes 36). De son imagination et de ses écrits émane une sorte de typologie de monstres où des antagonismes -amour et violence, bourreau et victime...s'amalgament, comme l'illustre l'extrait cité en exergue. Et les fréquentes métamorphoses «para-naturelles» de ses fictions s'avèrent quelque peu comme une projection de ses propres fantasmes d'altérité, comme elle l'affirme : «Je réagencerai mon A.D.N. afin de n'être plus celle que j'ai été. Je serai une mutante. Une hybride, comme je l'ai toujours revendiqué » (77).

C'est là une "mythologie personnelle» (36), certes. Mais cette focalisation sur l'hybride et le monstrueux ne serait-elle pas également une interrogation avec des notions bien présentes dans le contexte mauricien ? Dans le discours colonial par exemple, on retrouve une perception ambiguë et généralement problématique du phénomène du métissage - entre le monstre et le révolutionnaire (Vergès) -, allant jusqu'à prendre l'aspect d'un mixte diabolique, d'une difformité transgressive et déviante, le fruit d'une hybris

\footnotetext{
8 Par exemple le « Dossier spécial Ananda Devi» dans Notre Librairie 142 (2000) et dans Studia africana 19, Barcelona (2008), et le «Numéro spécial sur l'Océan Indien » de Nouvelles Études Francophones 23.1 (2008) quasi entièrement orienté sur l'écrivaine.

${ }^{9}$ « Puis-je changer de sexe et de corps? Ne garder de moi qu'une forme androgyne, asexuée ..., pourquoi ne pas tout détruire d'un seul coup en disant : je suis un monstre ?» (Devi, Les hommes 11).

10 « Les chiens et la mer, les monstres de mes rêves » (14).
} 
dangereuse et dérangeante à cause de son hétérogénéité insaisissable (Toumson 94). De nos jours, malgré une évidente revalorisation (et certaine commercialisation) de l'hybride, cette image négative du passé n'est pas entièrement révolue (Garcia et al.), et les écrivains mauriciens - y compris Devi - sont nombreux à interroger la question des identités plurielles et composites. D'une manière similaire, ils vont à l'encontre des logiques représentationnelles patriarcales qui ont pu attribuer aux femmes l'hystérie et la folie (Felman), construisant l'« excès du féminin » et la «monstruosité » comme pile et face d'une même médaille (Héritier 196-200).

Afin de lutter contre des constructions discursives qui instrumentalisent l'image du monstrueux pour ostraciser les identités autres'asociales', 'anormales', 'déviantes'-, certains écrivains s'approprient ce même trope. Dans leur posture contestataire et iconoclaste, ils ont en effet recours à l'usage du monstrueux balayant les bienséances et orthodoxies pour déclencher chez le lecteur une véritable prise de conscience. Car comme le dit David Gilmore, les travaux fondateurs de Mary Douglas à l'appui, les monstres exposent la perméabilité radicale et le caractère artificiel de toutes les frontières de nos systèmes de classification, de même qu'ils mettent en avant le caractère arbitraire et la fragilité de la culture ${ }^{11}$.

Ces réflexions préliminaires qui nous donnent déjà quelques pistes sur les subversions esthétiques chez Ananda Devi sauront informer nos interprétations de texte, particulièrement celles de son septième roman, La Vie de Joséphin le fou (2003), où l'ambivalence structurale de l'œuvre de l'auteure -le schisme entre 'monstruosité' et 'innocence' (Rochmann) $)^{12}$ — est portée à son paroxysme.

\section{La vie de Joséphin le fou (2003) ou le brouillage des repères}

Si La vie de Joséphin le fou semble avoir moins été l'objet d'analyses que d'autres romans de Devi, c'est peut-être en raison de l'aspect dérangeant du roman et à cause de son protagoniste masculin. Car il n'est aucun doute qu'il résiste aux lectures féminines/istes et identitaires plus évidentes des autres textes de l'auteure. Malgré les échos manifestes à l'œuvre entière de Devi — l'échec de la solidarité interhumaine et un dénouement pour les protagonistes dans la solitude et l'aliénation-, il y a une radicalité qui semble totale ici. Alors qu'on remarque chez d'autres héros de son univers littéraire (Paule, Anjali, Aeena, Ève...) une certaine évolution -quoique celle-ci s'opère par la transgression désespérée et la rébellion violente-, Joséphin va aussi loin, on le verra, que d'interdire toute forme d'évolution.

Après Pagli (2001) où l'héroïne Daya est stigmatisée par le surnom de 'Pagli', la 'folle', on retrouve de nouveau dans La vie de Joséphin le fou un personnage déviant et extravagant, cette fois-ci masculin et encore plus transgressif, car porteur de l'ambivalence 'innocence-monstruosité' e. L'intrigue insolite et à plus d'un égard hermétique se tresse en effet autour du

\footnotetext{
11 « Monsters expose the radical permeability and artificiality of all our classificatory boundaries, highlighting the arbitrariness and fragility of culture » (Gilmore 19).

12 Notons que Devi elle-même a fréquemment souligné cette double articulation 'innocentmonstre' qui structure son œuvre, par exemple lors des débats du colloque « Penser l'altérité : autour de l'œuvre d'Ananda Devi et des écrivaines mauriciennes contemporaines », Université catholique de Louvain, 30 Nov. 2007.
} 
protagoniste éponyme, un énigmatique jeune homme muet et (perçu comme) attardé qui, rejeté de son foyer, se métamorphose progressivement en une créature hybride amphibie qui hante les environs et devient le mythe dérangeant du pêcheur-nu, de l'homme-anguille, le fou Zozéfin-fouka. On remarque une autre intertextualité interne à l'hybridation de Moi, l'interdite (2000) où la protagoniste se mue en un chien. Dans sa (prétendue) démence et en manque d'amour, Joséphin enlève deux filles (Marlène, Solange) qu'il tient captives dans une grotte sous-marine. Mais incapable de communiquer et de plus en plus frustré par le monde et sa propre existence, il finit par tuer sa mère (Marlyn) et un de ses amants (les "tontons »), avant de massacrer, dans un acte de folie inconsciente - une sorte de geste sacrificiel - les deux filles. La narration très dense et à la première personne prend son dénouement cauchemardesque quand Joséphin se fait enfin dévorer par des anguilles.

C'est donc une histoire d'ostracisme, de fuite, d'isolement, de repli silencieux, de claustration, de refuge, comme on la trouve partout dans l'œuvre de Devi. Le protagoniste et sa mère font partie des « désemparés de la terre » (35) et deviennent des boucs émissaires : " autour de nous tout avait cette figure de haine et j'avais peur de tout»(18). Dans la chaîne des dominations et violences, la mère - rejetée par la famille et la communauté pour sa grossesse précoce et exploitée par les hommes- déverse sur l'enfant la frustration de son existence ratée, les punitions laissant sur son corps des traces telles des entrées d'un journal ${ }^{13}$. A côté d'un tel discours du corps aussi radical qu'abject, on retrouve aussi un autre leitmotiv de l'auteure, à savoir un rapport de genres désillusionné et une sexualité violente. Plusieurs scènes démythifient complètement l'acte sexuel et l'image récurrente d'un Eros associé à un Thanatos sombre et glauque annonce déjà le prochain roman, Ève de ses décombres (2006).

Avec les nombreux passages pour dire la dureté et l'échec de communication qui déterminent les relations humaines, l'anthropologie négative caractéristique des textes de Devi touche dans ce court roman à son comble. Le lecteur familier de la création littéraire contemporaine de l'île se dira donc : encore un enfant dérobé de son innocence, encore un "village au bout du monde» (32) qui a abandonné ses rêves, encore une société qui impose brutalement sa normativité, qui exploite les faibles et les marginaux, encore des hommes 'prédateurs' au phallus mortifère, encore partout des « salissures épouvantables » (74) et une isotopie extraordinaire de l'organique, de la putréfaction. Organes, déjections, viscosités, boue, corps disséqués et disloqués : le texte littéraire devient ici le reflet des théories de Mary Douglas (1996) et de Julia Kristeva (1980) sur la souillure et l'abject.

Or à la différence d'autres de ses romans, le déclin social, la rigidité religieuse, la sclérose morale ou le dépérissement humain sont à peine thématisés dans La vie de Joséphin le fou, mais apparaissent davantage comme des configurations métaphoriques. On relève par exemple un subtil jeu d'intertextualité à travers la berceuse "Larivyer Tanye", chanson au caractère hautement symbolique à Maurice. Mais l'émotivité et la signification identitaire qu'on connaît à la chanson sont déconstruites. Quand le

\footnotetext{
13 « Parfois ... elle venait inspecter mon corps et ma tête, toucher mon oreille qu'elle avait mordue un jour ... et comme ça elle explorait sa douleur sur mon corps, j'étais son livre d'histoire, elle se rappelait sa propre existence inutile dans mes plaies... » (Devi, La vie 21).
} 
protagoniste en fredonne des morceaux disparates, soulignant qu'il ne connait pas le reste et qu'on n'est jamais arrivé à le faire s'endormir, cela révèle toute de suite une rupture dans la filiation et une déchirure ontologique qui annonce son identité d'enfant non désiré dans un foyer monoparental, d'une femme devenue mère contre son gré à quinze ans.

Des configurations métaphoriques bien plus frappantes viennent à travers la mise en scène particulière de la $\mathrm{mer}^{14}$, et par détour, de l'identité de l'île elle-même. Devi n'échappe pas aux images exotisées du topos insulaire de la même façon que son homologue Barlen Pyamootoo ${ }^{15}$, mais elle transforme son protagoniste en un homme-poisson et l'envoie dans l'océan où semble régner encore une nature immaculée, juste, loin de la corruption humaine et de la violence du monde 'acculturé'. Depuis la mer, « le monde est changé » (20) et le retrait permet en effet de voir l'île d'une autre perspective qui la dénude dans toute sa laideur et mesquinerie. Ici la légèreté et le silence de l'eau, là les lourdeurs et les cris du " monde d'en haut » (20); d'un côté, la décadence, la tromperie, la dissimulation, la violence gratuite, le « misérable temps d'homme » (23) et « la sombre folie des hommes qui voient en permanence des ennemis » (72), de l'autre, la pureté, la franchise, le cycle de la vie et "l'océan qui travaille pour l'éternité» (23). La mer est chantée comme génitrice de l'île et des hommes $(46,47)$, lave et cicatrise les blessures $(19,20)$, devient même ersatz de mère $(22)$ et sanctuaire où tout se réduit à l'essence (21). Véritable leitmotiv de l'écriture féminine mauricienne, la mer a un rôle cathartique, abolit les cloisons identitaires, les affiliations normatives et exclusives, ainsi que les verrouillages communautaires. Par moments, on serait presque tenté de dire que l'archétype de la pastorale insulaire - Paul et Virginie (1788) de Bernardin de Saint-Pierre - est transféré ici au topos maritime. Joséphin y recouvre sa croyance tant innocente que fanatique en l'amour, jusqu'à y arborer des fantasmes de régression in utero dans un lieu où même le langage, autre site de son ostracisme, disparaît : "Pas de moqueries, sous la mer. Pas de mots. Pas de mots. Plus le temps passait et plus j'ai préféré me disparaître » $(23)^{16}$. En même temps, comme le dit l'écrivaine dans son récit autobiographique - «Mer et noyade sont synonymes» (Devi 2011: 36) - , c'est le caractère mortifère de la mer qui l'emporte. Celle-ci devient en effet ultimement le lieu de la dissimulation, de l'isolation et de l'exclusion et la fin du roman prend l'allure d'une nature qui, telle une divinité, demande le sacrifice ultime. Enfin, le symbolisme de la mer est avant tout plié à l'esthétique «para-naturelle» du roman ce qui doit mener à son élément le plus saillant, le protagoniste énigmatique.

\section{Ambivalences et étrangetés d'une figure hybride}

\footnotetext{
${ }^{14}$ Pour une lecture approfondie du symbolisme aquatique et marin dans Joséphin le fou, voir Bragard.

15 Une des particularités frappantes et 'désexotisantes' de Pyamootoo est en effet de détourner le regard de l'île en situant l'action de son roman Bénarès (1999) quasi exclusivement la nuit et en délocalisant l'intrigue du Tour de Babylone (2002) en Irak.

${ }^{16}$ La curieuse forme pronominale est récurrente chez Devi et se retrouve aussi dans Moi l'interdite dans le propos de la protagoniste : « Ils me laissaient dans ce four à chaux quand il fallait me disparaître » (Devi, Moi 33).
} 
Joséphin est un personnage 'anormal', 'déviant' et ambigu qui se transforme en figure transgressive et schizophrénique. À trois ans, il subit un traumatisme crânien par un des amants de sa mère et ne parle plus. Cela est quelque peu évocateur d'Oskar Matzerath, le jeune héros du Tambour (1959) de Günther Grass - roman entrecoupé, on le sait, par plusieurs éléments fantastiques et surréalistes - qui, suite à une chute (volontaire) à l'âge de trois ans également, interrompt le processus naturel de sa propre croissance et s'arrête de parler tout en s'énonçant d'une conscience d'adulte qui le fait devenir un fervent critique des tares sociétales. Stigmatisé pour ses défaillances de communiquer, perçu comme « un gaga » et « un retardé » (35), Joséphin s'enfuit pour devenir une sorte de figure mythique et hanter les lieux " comme une ombre, un fantôme, un monstre, Joséphin le fou, Zozéfinfouka » (38). Sa métamorphose vient donc comme réaction et prolongation d'une stigmatisation extérieure. Expulsés par la société, le monstrueux et l'abject reviennent avec vengeance. Si le motif central de la déréalisation touche aussi d'autres personnages du roman autour desquels se tisse toute une isotopie de la lente désagrégation qui va de l'auto-abandon à la zombification, de l'« inhumain » à l' « innommable » (34), de la dilution (39) au « vide » (33, 82), c'est Joséphin qui est le pivot de cet univers de l'insolite et de l'invraisemblable.

Il est perçu comme « un petit animal qui a vite cessé de prononcer des mots, sauf dans sa tête » (40) et il est symptomatique qu'on le prenne dans une scène du roman pour un chien, tellement il est sale, maigre et recroquevillé dans son coin (36-37). Rejeté par les humains, c'est la mer qui entre en lui et devient son élément. Mais comme s'il fallait un rite de passage d'un univers à l'autre, une transformation s'impose qui arrive sous forme d'anguilles ${ }^{17}$ qui, pendant le sommeil du héros, le couvrent, explorent et prennent possession de son corps au point de fusionner avec lui. Si cet étrange acte évoque d'autres romans de l'auteure - les insectes qui se repaissent de la protagoniste Mouna dans Moi l'interdite (2000), l'image des insectes dévorants étant déjà reprise de L'Arbre-fouet (1997) - Devi déploie tout son lyrisme charnel pour décrire minutieusement cette scène nauséabonde $(24,50-51)$. Le narrateur abandonne donc son humanité pour passer non pas dans un au-delà, mais pour se muer littéralement en une créature hybride et monstrueuse.

On ignore s'il est davantage homme qu'animal, s'il est mort ou vivant. Sa transformation ichtyo-morphique est aussi une aliénation progressive et il finit par se décrire lui-même comme « un cadavre ambulant» (81). La phrase aussi opaque qu'iconique, «j'ai préféré me disparaître »(23), se réalise enfin pleinement dans le rituel morbide final. Dans un excipit qui rappelle le fameux Parfum (1985) de Patrick Süskind - où la mégalomanie du protagoniste JeanBaptiste Grenouille, également un être mystérieux, obsessionnel et doté de pouvoirs surhumains, s'achève en apothéose abjecte en se faisant littéralement dévorer par amour par ceux qui sont tombés sous son envoûtement -, Joséphin livre les filles massacrées aux requins avant de se faire engloutir lui-même par les anguilles.

On ne sait donc pas vraiment qui est Joséphin, si ce n'est le fait que le monde réel ne sait saisir son identité. Cela dit, rappelons une banalité : il est le

\footnotetext{
${ }^{17}$ Voilà un autre parallèle avec Le Tambour de Grass où une pêche aux anguilles —avec une
} tête de cheval- devient une des scènes les plus marquantes et abjectes du roman. 
narrateur. Donc l'étrangeté de la diégèse et de l'effet de lecture interroge le lecteur. Doit-il croire tout cela ? Que faire d'une telle métaphorisation ? Est-il un narrateur digne de confiance ? Où est la frontière entre rêve et réalité ? Qu'est-ce qui est fabulation et mensonge ? Comment séparer l'obsession du discours lucide et fiable? Un tel récit n'est-il pas que mirage psychédélique, hallucination d'un fou ? Entre construction et destruction, on n'est plus sûr et le lecteur est perdu lui-même dans la conscience d'un personnage quasi schizophrène. L'irruption de la troisième personne dans un roman à la première personne en serait son expression narratologique. Enfin, Devi refuse, comme toujours, la fermeture et le cadre narratifs par une instance auctoriale bien ancrée qui inspirerait de la confiance. Le dénouement du récit par un narrateur qui se fait dévorer et la clôture existentielle totale constituent le coup de cymbale final d'un texte où tous les repères se perdent, notamment pour le lecteur.

Quel sens doit-on alors donner à ce personnage aliéné, aussi innocent que monstrueux, aussi rédempteur qu'ange diabolique? Bien évidemment, Joséphin est d'abord une existence brisée, victime des cloisons et des hypocrisies sociales et humaines, rejeté, car 'illégitime', et en raison de son handicap, de sa 'déviance' physique. En même temps, il se fait bourreau abject, et cela non seulement par 'juste' vengeance. Dans le cas de sa mère et d'un de ses amants, le meurtre de la mère peut être compris comme un acte d'amour et de grâce, un geste "euthanasique" qui la délivrerait d'une existence invivable tandis qu'il se délivre lui-même de la culpabilité de n'avoir jamais su la combler (82). Or ce qui importe davantage c'est qu'il devient châtieur par hybris, par jalousie et amour propre blessé, comme dans le cas des deux filles. Si son exclusivité s'est manifestée bien avant - il éprouve de la Schadenfreude quand il casse les casiers et coupe les lignes des pêcheurs, car il considère la mer comme son terrain, son royaume-, la manie est poussée au paroxysme avec Marlène et Solange. Son idolâtrie est aveugle (« si jolies si gentilles même lorsqu'elles vomissent de me voir », 83) et sa fatuité complète (« la beauté que moi seul peux leur donner»,83). Il leur voue un fétichisme et une adoration fanatique qui se transforme en possessivité mégalomane. Enfin, le meurtre n'est pas un accident, mais une sorte de sacrifice d'extrême cruauté dont la description investit tous les registres du monstrueux et de l'horrible : d'abord dans une chaine de rêves annonciateurs sanglants, puis dans sa réalisation inavouée, l'apogée de l'abject :

Deux poupées brisées avec une brutalité de bête. Bras jambes en désordre, postures impossibles. Un os luit, clair, nettement déboité ... Corps désacrés, massacrés, font eau de toutes parts. Font sang de toutes parts. Le sang a jailli et a giclé sur les parois de la cave, auréole leurs cheveux glués, maquille de rouge leurs bouches dévorées. Pénétrées, profondément, par la mort. Transpercées par sa présence, par son aiguille. La mort est entrée ici, je sais pas comment... (86)

Avec un assassin qui ne sait plus comment il a tué ses victimes, le brouillage entre rêve et réalité, phobies et fantasmes, bien et mal est total. Il est bien difficile de voir l'idée de l'« innocence meurtrière », avancée dans le quatrième de couverture et l'ambiguïté est à son comble. Le monde devient un univers étrange où la précarité et la dureté de l'existence deviennent les seules 
certitudes. Et le processus de déréalisation est intensifié par une violence langagière caractéristique de l'écriture de l'auteure.

Comment alors donner crédit au discours social et démystificateur du texte ? Comment évaluer cette représentation non seulement dégrisée, mais aussi complètement déréalisée du topos insulaire et de ses identités ? Quelle signification doit-on lire dans cette figure ambivalente et de ses actes monstrueux?

\section{Des apories identitaires à l'incapacité de communication}

Malgré le caractère hermétique de ce bref roman dont l'esthétique ne se prête pas à une lecture facile, il est possible d'avancer un certain nombre d'approches interprétatives. D'abord, une telle mise en scène donne à lire un lieu qui est investi par l'image de l'enfermement et de la claustration — «le départ impossible de cette île » (33) - , et qui dépasse l'image bien connue de l'île-prison. Le narrateur parle d'un espace instable et incertain, toujours sous la menace de disparaître : " la mer, elle a qu'à sortir la langue un jour, sans trop se fatiguer, elle a qu'à lécher l'île de cette langue paresseuse et en un rien de temps, elle l'aura ramenée d'où elle vient. Histoire terminée » (46). Attribuer ces paroles à un prêcheur dément déversant ses fantasmes morbides et mélancoliques sur la fin du monde est tentant, mais clairement insuffisant. Car il n'est question ici de rien de moins que de la vie, ou plus précisément du 'droit' à la vie. Et c'est donc ici que le topos insulaire rejoint par la thématique majeure du texte, à savoir celle de la normativité.

Joséphin est un poids, pire une tare pour ceux comme sa mère qui veut un « enfant normal » (25) et désire « vivre normale[ment]» (40). Par ailleurs, elle-même, franchit, par extension, le seuil du socialement acceptable, du tolérable, du 'normal'. Faire irruption avec sa présence dérangeante dans ce monde des catégories, des identités clairement assignables mène alors au " complot du rejet» (40), dont le lecteur lui-même, dans un court passage phatique et très puissant, devient complice, comme le dit le protagoniste : «Ricanez pas, vous aussi avez fait de Joséphin, dans votre petite tête ordonnée, un fouka qu'il faut rayer des souvenirs » (40). Il touche ici un point sensible, ralliant le lecteur à la communauté des conspirateurs qui, dans une économie de rituel expiatoire, purgeraient leur intégrité de groupe de la souillure. Considéré comme un pestiféré et rejeté, Joséphin ne doit pas en effet «franchir la ligne qui [le] sépare des vivants» (83). Dans l'imaginaire collectif, comme l'affirme justement Magdelaine-Andrianjafitrimo («J'ai préféré » 262), il n'est acceptable que comme une créature surnaturelle, comme une légende. Vu ainsi, le texte touche à des notions aussi substantielles que l'humanité, l'existence, le vivant, la légitimité, la normalité... et peut se lire à un écho écho artistique aux conceptualisations et théories de Judith Butler. Avec Joséphin, plus encore qu'avec ses autres personnages 'déviants', Devi sème le «trouble», et son protagoniste est en effet un spécimen particulièrement saillant de ces "êtres socialement "impossibles", illisibles, irréalisables, irréels et illégitimes » (Butler, Trouble 26) qui ne se laissent pas concevoir en termes stables ou permanents. On notera par ailleurs que les ouvertures conceptuelles de la philosophe américaine -élargissant son travail sur le genre à la notion d'humain pour s'interroger plus généralement sur le fait de savoir (ce) qui est considéré comme 'légitime', 'normal' et 'réel' dans 
l'espace public ${ }^{18}$ - constituent également une grille d'interprétation fructueuse pour le texte de Devi.

Il est certes vrai que dans La Vie de Joséphin le fou, la transgression de 1' 'humain' orthodoxe et conventionnel va loin et que Devi pousse la question de l'altérité à son paroxysme. Que les identités deviennent complètement incommensurables et intraduisibles se montre notamment dans la scène où, dans sa nudité, sans parole et en faisant une sorte de danse étrange, Joséphin se présente aux filles qui (tout logiquement) s'effraient et se referment, au grand dam du héros qui se résigne ainsi : " je suis normal, créature naturelle, mais elles, elles avaient pas l'habitude, couvertes qu'elles étaient tout le temps...» (57). Un tel échec de communication montre sans aucun doute la limite du partage des identités et semble significatif sur le plan littéraire. Ici, les mondes ont perdu leur dénominateur commun, les univers se sont complètement détachés les uns des autres. Cela étant dit, au-delà de telles apories, l'esthétique particulière du roman fait de lui certainement aussi un objet privilégié de réflexion sur l'éthique, et ce malgré l'étrangeté du texte et l'ambiguïté du protagoniste qui rendent souvent très difficile l'adhésion du lecteure.

Enfin, l'introduction de la question éthique n'empêche pas Devi dans précisément cette logique d'incommensurabilité- de diriger sa construction romanesque vers le néant. Si certains éléments du roman suggèrent une prise de parole postcoloniale à la fois critique et constructrice - la « longue longue mémoire » (24) dénonciatrice du narrateur et ses beaux rêves en seraient des exemples ${ }^{19}$-, tout finit dans la désillusion la plus complète. Son utopie est inatteignable et vire au désastre. Avec un dénouement aussi violent et un tel manque de perspective, l'intrigue tend en effet vers une vacuité quasi ontologique qui ne prendrait sens que dans une sorte de prophétie apocalyptique.

Ainsi, tout d'abord, Joséphin semble n'accorder presque aucun crédit à l'humanité sur la voie du déclin ainsi. De plus, le refus des seules personnes à pouvoir lui donner du sens radicalise cette posture. Le dialogue reste irréalisable et les écarts impossibles à s'amoindrir. Il a beau avoir le projet 'noble' de sauver les filles « de la lourdeur de la terre » (59) et notamment du lugubre chemin tracé des femmes 'ordinaires' dans un passage à la verve féministe lyrique, mais la sublimation de ses « reines » (32) ou " princesses » $(9,31,60,80)$ annule en même temps leur subjectivité et leur vole le libre arbitre. Car aussi " glorieuses » qu'elles soient, Marlène et Solange restent des " prises » (32), c'est-à-dire qu'elles s'inscrivent dans le paradigme (patriarcal et misogyne) de la chasse et de la captivité. Quoique pur dans son cœur n'est-il pas en permanence à la recherche de «quelques particules d'amour »

18 Voir par exemple sa collection d'interviews au titre révélateur -Humain, inhumain. Le travail critique des normes (2005) — où Butler s'appuie sur la conceptualisation de Giorgio Agamben sur le «homo sacer» - ces personnes apatrides, liminales et non reconnaissables comme vivantes - afin de s'interroger sur «la définition contingente de l'humain » (50), c'est-à-dire ce qu'elle exclut et a exclu afin de parvenir à la cohérence qu'elle présente. Butler ne parle pas de personnes et de groupes «simplement oblitérés, ou effacés, ou juste rendus invisibles », mais plutôt de ceux qui, malgré leur présence et visibilité sont "présents sur un mode ontologiquement suspendu » (51).

19 Voir par exemple : «...rêve d'accoucher d'une île plus large, moins épineuse pour ces enfants de nulle part et d'ailleurs, ceux qui n'ont aucun coin à eux, aucun lieu et aucune demeure... » (Devi, La vie 72). 
(72) laissées par sa mère-, il ne fait que reproduire la réification et la possessivité des hommes dans l'île. Autrement dit, s'il déconstruit avec lucidité les chimères et les aveuglements conscients des hommes, il n'est pas à la hauteur de ces mêmes exigences et succombe à sa fatuité et son incapacité à séparer fantasme et réel. Son désir de possessivité — « je suis leur seul espoir, je suis leur sauveur, je suis leur vie!»(62) - s'avère comme une hybris totale. Quand il se rend enfin compte d'une part que « protéger [les filles] de la vie est impossible»(43), de l'autre, qu'il reste incompris — « elles ont pas compris. Vilain. C'est tout ce que je suis » (70)—, sa manie et misanthropie se durcissent. Rejeté par ceux qui protègent leur existence par le biais de l'essentialisme et l'exclusion, il s'évade lui-même dans des essentialismes qui ne sauront plus le réconcilier avec les hommes. C'est le fanatisme qui transforme l'amour en mort, la beauté en souillure, la tendresse en cruauté. Dans un tel brouillage de valeurs et de repères, il n'est pas surprenant que le massacre des filles implique aussi leur viol — « La mort est entrée ici ... est entrée en elles dans leurs cuisses écartées » (86)_, conférant au leitmotiv phallique de l'anguille une signification mortifère supplémentaire. Et contrairement au dénouement de Moi l'interdite qu'on peut considérer comme le miroir de La Vie de Joséphin le fou et où la protagoniste métamorphosée finit par se reconnecter avec l'humanité, l'exclusion et la violence chez Joséphin restent totales. Quand le désir paroxystique de fusion ne peut être assouvi, c'est celui de la dissolution qui l'emporte. Le dénouement radical du roman, en écho aux intimités de l'auteure partiellement dévoilées lorsqu'elle évoque une rupture personnelle, est alors « une voie empruntée parmi les monstres [qui] est sans retour » (Devi, Les hommes, 171).

Le destin réservé aux personnages de Devi est irrévocable et les ressemblances intertextuelles avec d'autres figures littéraires plus ou moins étranges s'arrêtent là. Chez Grass, Oskar reste certes séparé du monde des adultes 'normaux' et est injustement interné dans un asile psychiatrique, mais il garde son esprit critique, écrit ses mémoires et s'apprête à être libéré. Et si Grenouille dans Le parfum finit par être dépecé dans un acte auto-sacrificiel similaire à celui de Joséphin, cette anthropophagie mégalomane prend quelque peu l'allure d'une fusion amoureuse, car il est pris pour un ange et s'est rendu, grâce à son parfum parfait, 'identique' aux hommes. Le héros chez Devi en revanche est dévoré précisément parce qu'il est enfin transformé en un « corps étranger, blessé, immobilisé » (87), parce qu'il est devenu méconnaissable même aux créatures du monde maritime.

Ainsi, non seulement, parmi les hommes, aucun espoir n'est possible, aucune rédemption n'est en vue, la souillure est trop complète, mais également parmi les éléments. Dans La Vie de Joséphin le fou, on attend en vain la perspective d'une purge cathartique, d'un univers parallèle d'où renaitrait des cendres un phénix. Finalement, la question éthique centrale du vivant - le reproche de sa mère : "mais tu es là vivant, enfin vivant plus ou moins..." (25) et sa propre interrogation : " mais c'est quoi, être vivant? Je sais encore pas » (39) — n'aura pas trouvé de réponse. L'idée d'un " monde neuf » (79) doit rester l'utopie. Et, de plus, l'utopiste lui-même se désagrège. Chez Devi, la déréalisation est complète et s'achève en apothéose monstrueuse. Elle s'est substituée au fur et à mesure à la réalité pour enfin se fondre dans le néant. La mutation minérale ou animale qui pourrait paraître comme une fin sacrificielle et expiatoire ne symbolise aucun retour vers l'origine du monde. Ce qui reste 
comme «l'aboutissement d'un parcours contre-initiatique » (Delmeule 119), c'est la cruauté, la dislocation des êtres, l'effacement total : « je ne sens rien, non, rien » (Devi, La vie 88) sont aussi les dernières paroles d'une figure dissolue dans un absolu monstrueusement effrayant.

\section{Conclusion}

La vie de Joséphin le fou est un texte à la voix incandescente, viscérale, vibrante de rage, qui traduit un langage du corps, charnel et sulfureux mais aussi obsessionnel et souvent abject. Le lecteur est face à un discours transgressif, oral, fragmenté, elliptique, introspectif, incohérent, chaotique, polyphonique qui non seulement reproduit la déchirure du protagoniste, mais qui cherche les limites de la prose romanesque. Le roman suscite sans aucun doute des effets de lecture particulièrement étranges et une très grande difficulté d'identification de la part du lecteur qui est laissé devant des contradictions troublantes et des apories d'un personnage énigmatique et d'un texte fuyant qui résistent à la clôture interprétative.

Si les « créatures de l'ambiguïté » (Zuckerman) sont sans aucun doute une des marques de la plume de Devi, ses scénographies de l'étrange s'inscrivent de manière générale dans l'entreprise postcoloniale du roman mauricien contemporain de faire éclater espaces et imaginaires. Si elle n'est pas la seule à mettre en place une esthétique de l'《anti-tropicalisation », de l'étrange, de la déréalisation ainsi qu'une certaine anthropologie négative d'où une réelle intertextualité interne perceptible dans cette jeune production romanesque de l'île-, elle est la seule à aller aussi loin dans la création des figures de l'altérité, de l'abject et du monstrueux, à pousser le caractère conflictuel de la déviance et de l'hybridité à ses limites.

Avec ce protagoniste insolite, sa morphologie 'anormale' et 'monstrueuse' et sa déviation radicale des normes sociales, on peut certes se demander avec Butler (Trouble 245) quelle est la puissance d'action en société de ces figures inintelligibles, au raisonnement opaque et aux corps désintégrés. Mais ce serait trop recourir aux logiques correspondant à une esthétique réaliste, croyant en l'illusion d'un impact sur le monde et les choses, car l'étrangeté, la déréalisation, la monstruosité sont autant d'éléments qui soulignent chez Devi «l'échec d'un discours réaliste qui chercherait à explorer le monde pour avoir sur lui une action réformatrice » (MagdelaineAndrianjafitrimo, «J'ai préféré » 256). En même temps, son imaginaire singulier nous permet de forer dans l'indicible, de tâter les non-dits et les espaces-temps interstitiels, et de nous interroger de manière (auto)critique sur le réel.

Avec les représentations violentes et paroxystiques de l'auteure, son univers «para-naturel» et son écriture intense amalgamant incantations lyriques et rage brute, nous ne sommes pas dans des propositions sensationnalistes et gratuites qui esthétiseraient la violence ou en feraient un spectacle. Elles ne tiennent pas non plus de la caricature, du rocambolesque ou du fantasque. L'étrangeté, l'hybridité, la liminalité et la monstruosité mises en scène peuvent devenir momentanément vectrices d'une déconstruction critique qui fustige les cloisons identitaires et la dégradation humaine. Elles incitent à réfléchir sur la construction des identités, de la déviance et de la marginalité, à remettre en question les fonctionnements des taxonomies oppressives et à 
dévoiler les diktats du regard social avec ses divisions et hiérarchies. Mais elles interrogent surtout les normativités et posent la question éthique par excellence, celle de l'humain et du vivant. Le jeu sur les ambiguïtés, ambivalences et les liminalités dit donc au final la quête identitaire de l'individu 'subalterne' et 'mineur' des univers postmoderne et postcolonial, la lutte contre le rejet de l'altérité et l'engagement pour ces figures qui ont « déjà abandonné la vie» (Devi, La vie 51). Et forcément, dans la société mauricienne avec ses relations complexes entre appartenances et différences, une telle exploration littéraire d'identités hybrides, ambiguës et liminales prend donc une signification aussi poétique que politique.

Que ces interrogations finissent par être canalisées vers des apories, des incommensurabilités et un vide ontologique tel que nous avons du mal à en trouver ailleurs dans le roman mauricien doit bien évidemment paraître destructif. Or chez Devi, une telle négativité n'est enfin rien d'autre que le résultat d'une incapacité à se faire entendre, d'une impossibilité à dialoguer avec les hommes, comme le dit le protagoniste après les vaines tentatives de sa mascarade séductrice : "Pourquoi on a tant de mal à se comprendre ? Sans mots, et même avec des mots, c'est impossible » (58). Peu importe la configuration métaphorique qu'il sert, un tel énoncé dans une œuvre fictionnelle n'est bien évidemment jamais innocent. Ce qu'il traduit chez Devi, c'est la monstruosité de l'incommunication : la déréalisation fait ici le saut de l'esthétique au poétique et méta-communicatif et devient en fin de compte dispositif pour sonder les possibilités et les limites de la prise de parole ainsi que du discours littéraire. 


\section{Bibliographie}

Alexis, Jacques Stephen. « Du réalisme merveilleux des Haïtiens. » Présence Africaine, vols. 8-10, 1956, pp. 245-271.

Arnold, Markus. La littérature mauricienne contemporaine. Un espace de création postcolonial entre revendications identitaires et ouvertures interculturelles. Berlin: Lit, 2017.

--- «Les univers étranges, intimes et violents de l'auteur mauricien Carl de Souza : un cas d' "anti-tropicalisation" du roman. » Les Lettres Romanes, vol. 68, n¹-2, 2014, pp. 73-101.

Ashcroft, Bill, Gareth Griffith, et Helen Tiffin. The Empire Writes Back. London and New York: Routledge, 1989.

Bernabé, Jean, Patrick Chamoiseau, et Raphaël Confiant. Éloge de la Créolité. Paris: Gallimard, 1993.

Bloch, Henriette, et al. Dictionnaire fondamental de la psychologie. A-K. Paris: Larousse, 1997.

Bozzetto, Roger. « Littérature, émerveillements et critique littéraire. » Démons et merveilles. Le surnaturel dans l'Océan Indien. Eds. Valérie Magdelaine-Andrianjafitrimo, Jean-Claude Carpanin Marimoutou et Bernard Terramorsi. Saint Denis: Université de La Réunion, 2005: 2539.

Bragard, Véronique. "L'enfant des vagues ou de la vase: la symbolique marine dans La Vie de Joséphin le fou d'Ananda Devi. » Women in French Studies, vol.15, 2007, pp. 84-97.

Butler, Judith. Trouble dans le genre. Le féminisme et la subversion de l'identité. Traduit par Cynthia Kraus. Paris: La Découverte, 2005.

---. Humain, inhumain. Le travail critique des normes. Entretiens. Traduit par Jérôme Vidal et Christine Vivier. Paris: Éditions Amsterdam, 2005.

Chakrabarty, Dipesh. Provincializing Europe: Postcolonial Thought and Historical Difference. Princeton and Oxford: Princeton UP, 2008. Print.

Damlé, Amaleena. "Towards a Poetics of Reconciliation: Humans and Animals in Ananda Devi's Writing. » International Journal of Francophone Studies, vol.15, n³-4, 2012, pp. 497-516.

Delmeule, Jean-Christophe. «La Vie de Joséphin le fou ou la dislocation du récit. " Studia Africana, vol. 19, 2008, pp. 117-120.

Devi, Ananda. Moi, l'interdite. Paris: Dapper, 2000.

---. La vie de Joséphin le fou. Paris: Gallimard, coll. «Continents noirs », 2003.

---. Les hommes qui me parlent. Paris: Gallimard, 2011.

Douglas, Mary. Purity and Danger: An Analysis of Concepts of Pollution and Taboo. London: Routledge and Kegan Paul, 1966.

Écritures mauriciennes au féminin : penser l'altérité. Éds. Véronique Bragard et Srilata Ravi. Paris: L'Harmattan, 2011.

Felman, Sander. «Women and Madness: the Critical Phallacy. " Diacritics vol. 5.4, 1975, pp. 2-10.

Galibert, Nivoelisoa. "Quelques enjeux littéraires et stratégiques de la diaspora indianocéanique francophone : l'exemple d'Ananda Devi. » Ed. Julia Waters. e-France: an on-line journal of French Studies, vol. 2, 2008, pp. 75-99. 
Garcia, Mar, Felicity Hand et Nazir Can, éditeurs. Indicities/Indices/Indícios. Hybridations problématiques dans les littératures de l'Océan Indien.. Ille-sur-Têt: Éditions K'A, 2010.

Gilmore, David D. Monsters: Evil Beings, Mythical Beasts, and All Manner of Imaginary Terrors. Philadelphia: University of Pennsylvania Press, 2003.

Héritier, Françoise. Masculin-féminin. I : La pensée de la différence. Paris: Odile Jacob, 1996.

Issur, Kumari. "Psychopathologies dans l'œuvre d'Ananda Devi. » Les représentations de la déviance. Ed. Corinne Duboin. Paris: L'Harmattan, 2005: 203-208.

Kistnareddy, Ashwiny O. Locating Hybridity: Creole, Identity and Body Politics in the Novels of Ananda Devi. Bern: Peter Lang, coll. " Modern French Identities », 2015.

Kristeva, Julia. Pouvoirs de l'horreur. Essai sur l'abjection. Paris: Seuil, 1980.

Le Bris, Michel et Jean Rouaud, éditeurs. Pour une littérature-monde. Paris: Gallimard, 2007.

Magdelaine-Andrianjafitrimo, Valérie «"J'ai préféré me disparaître" ou la dissolution du réel mauricien chez Ananda Devi, Shenaz Patel, Nathacha Appanah-Mouriquand. » Dérives et déviances. Ed. Corinne Duboin. Paris: Le Publieur, 2005, pp.255-264.

---. "Les égarements du réel dans Les Jours Kaya de Carl de Souza.» Démons et merveilles. Le surnaturel dans l'Océan Indien. Eds. Valérie Magdelaine-Andrianjafitrimo, Jean-Claude Carpanin Marimoutou et Bernard Terramorsi. Saint Denis: Université de La Réunion, 2005, pp. 433-458.

Marimoutou, Jean-Claude Carpanin. «Littératures indiaocéaniques. » Revue de littérature comparée, vol. 318, $\mathrm{n}^{\circ} 2,2006$, pp. 131-140.

Marson, Magali. «Carnalité et métamorphoses chez Ananda Devi. » Notre Librairie, vol. 163, 2006, pp. 64-69.

Piéron, Henri. Vocabulaire de la psychologie. Paris: PUF, 1951.

Ramharai, Vicram. "Solstices, univers ambigu ou réalisme magique ?" Démons et merveilles. Le surnaturel dans l'Océan Indien. Eds. Valérie Magdelaine-Andrianjafitrimo, Jean-Claude Carpanin Marimoutou et Bernard Terramorsi. Saint Denis: Université de La Réunion, 2005, pp. 459-471.

Rochmann, Marie-Christine. "Monstruosité et innocence dans La Vie de Joséphin le fou. » Nouvelles Études Francophones, vol. 23, nº 1, 2008, pp. 163-174.

Sultan, Patrick. « Ruptures et héritages. Entretien avec Ananda Devi. » Orées, vol. 1, n², 2001, www. http://orees.concordia.ca/. Consulté le 30 août 2013. Indisponible.

Terramorsi, Bernard. «Le fantastique et les littératures de l'océan Indien : introduction à une recherche. " L'océan Indien dans les littératures francophones, Pays réels, pays rêvés, pays révélés. Eds. Kumari Issur et Vinesh Y. Hookoomsing. Paris: Karthala, 2001, pp. 167-178.

---. «Préface. Le surnaturel dans l'océan Indien: les archipels de la différence. » Démons et merveilles. Le surnaturel dans l'Océan Indien. Eds. Valérie Magdelaine-Andrianjafitrimo, Jean-Claude Carpanin 
Marimoutou et Bernard Terramorsi. Saint Denis: Université de La Réunion, 2005, pp. 5-10.

Toumson, Roger. Mythologie du métissage. Paris: PUF, coll. « écritures francophones », 1998.

Tyagi, Ritu. Ananda Devi: Feminism, Narration and Polyphony. Amsterdam and New York: Rodopi, 2013. Print.

Vergès, Françoise. Monsters and Revolutionaries: Colonial Family Romance and Métissage. Durham: Duke University Press, 1999.

Zuckerman, Jeffrey. " Creatures of Ambiguity (interview with Ananda Devi). »Los Angeles Review of Books. 5 mai 2015. https://lareviewofbooks.org/article/creatures-of-ambiguity-a-qa-withananda-devi\#!. Consulté le 19 janvier 2018. 